\title{
O PANORAMA DAS ATIVIDADES NÃO PRESENCIAIS RELATIVAS AO PRÉ-CÁLCULO E CÁLCULO I NO IFNMG - CAMPUS MONTES CLAROS
}

DOI: $10.37702 / 2175-957 X . C O B E N G E .2021 .3610$

Éllen Caroline Costa Santa Rosa - eccsr@aluno.ifnmg.edu.br Instituto Federal do Norte de Minas Gerais

Rua Dois 300

39404-058 - Montes Claros - MG

Daniel Leite Mesquita da Silva - dlmds@aluno.ifnmg.edu.br

Instituto Federal do Norte de Minas Gerais

Rua Dois 300

39404-058 - Montes Claros - MG

André Vinícius Mendes Barros - mb.andrevinicius@gmail.com

IFNMG

Itajaí 190

39404-225 - Montes Claros - MG

Tatiane Reis do Amaral - tatianeramaral@gmail.com

IFNMG

Avenida olimpio Prates 1200

39403-261 - Montes claros - MG

NEILA MARCELLE GUALBERTO LEITE - neila.gualberto@ifnmg.edu.br INSTITUTO FEDERAL DO NORTE DE MINAS GERAIS

RUA TEJO 45

39408-121 - MONTES CLAROS - MG

Resumo: Muitos alunos ingressam no ensino superior com expressivas lacunas em relação ao conhecimento matemático (GOMES 2012). Percebe-se que muitos conceitos foram compreendidos de forma equivocada, o que dificulta e, às vezes, impede a apropriação de novos conhecimentos. O Cálculo Diferencial e Integral são disciplinas com alto índice de reprovação nos cursos do IFNMG-Campus Montes Claros, assim como em várias instituições. $O$ baixo rendimento nessas disciplinas 
acabam causando um desânimo nos discentes levando, muitas vezes, à evasão do curso. A falta de base em matemática elementar contribui expressivamente com esse cenário. Baseado no exposto, faz-se necessário o desenvolvimento de projetos e programas que visem o combate à repetência e evasão nessas disciplinas, visando colaborar com a permanência e êxito do aluno no curso, além de estabelecer conhecimentos sólidos de base matemática para seu sucesso no curso. Neste trabalho, apresentamos um panorama geral do curso de Pré-Cálculo seguido da disciplina Cálculo I ANP, ofertada aos ingressantes nos cursos de Engenharias e Ciência da Computação do IFNMG-Campus Montes Claros em 2020, bem como uma breve análise do desenvolvimento do ensino e seus resultados. Refletimos, ainda, sobre novos caminhos a seguir no intuito de melhorar as próximas ofertas desta disciplina.

Palavras-chave: Ensino. Atividades não presenciais. Cálculo. Matemática básica. 


\section{O PANORAMA DAS ATIVIDADES NÃO PRESENCIAIS RELATIVAS AO PRÉ-CÁLCULO E CÁLCULO I NO IFNMG - CAMPUS MONTES CLAROS}

\section{INTRODUÇÃO}

A evasão escolar, tanto no ensino básico quanto no superior, é um fenômeno que sempre existiu (Gaioso 2005, Bardagi 2007). No entanto, desde que passou a figurar entre os indicadores da planilha de alocação de recursos para as Instituições Federais de Ensino Superior (IFES), tornou-se alvo das políticas públicas e de ações, visando a permanência e êxito do discente. Assim, várias pesquisas têm sido desenvolvidas no sentido de compreender e combater este problema ou, pelo menos, amenizá-lo (Silva Filho et al 2007, Giliolli 2016).

Nos cursos de graduação, muitos fatores influenciam na evasão escolar tais como a forma de ingresso, falta de afinidade com o curso, falta de perspectiva com relação à profissão e entre eles podemos ressaltar a dificuldade em algumas disciplinas do curso (Ambiel 2015, Wrobel et al 2013). Um dos motivos da evasão nos cursos de Ciência da Computação e de Tecnologias pode estar vinculado à falta de domínio dos conhecimentos matemáticos e ao baixo rendimento dos alunos em Matemática no ensino básico (Barcelos \& Silveira 2012).

O Cálculo Diferencial e Integral é uma disciplina que faz parte de todas as matrizes curriculares dos cursos de Ciências Exatas e Engenharias. Suas exigências de pensamento abstrato para a compreensão de novos conceitos, além das expressivas lacunas em relação ao conhecimento matemático que os alunos trazem consigo do ensino básico (Gomes 2015) são os principais fatores que colaboram com esta conjuntura. Percebe-se que muitos conceitos foram compreendidos de forma equivocada, o que dificulta e, às vezes, impede a apropriação de novos conhecimentos. Um agravante para esta questão vincula-se ao fato de que os alunos, em grande maioria, desconhecem essas lacunas (Kessler 2013).

O Cálculo Diferencial e Integral é uma disciplina com alto índice de reprovação nos cursos do IFNMG Campus Montes Claros, assim como em várias instituições de ensino superior. O baixo rendimento nessas disciplinas acabam causando um desânimo nos discentes levando, muitas vezes, à evasão do curso (Barcelos \& Silveira 2012).

Diante deste cenário, faz-se necessário o desenvolvimento de projetos e programas que visem o combate à repetência e evasão nessas disciplinas, visando colaborar com a permanência e êxito do aluno no curso, além de estabelecer conhecimentos sólidos de base matemática para seu sucesso no curso. Baseado no exposto, como o escopo deste trabalho, buscou-se contribuir efetivamente com a melhor formação dos discentes ingressantes nos cursos de Engenharia Química e Ciência da Computação do Instituto Federal do Norte de Minas Gerais - Campus Montes Claros, ao implementar uma proposta de curso de Pré-Cálculo, baseado em conteúdos do ensino fundamental e médio, para preencher expressivas lacunas de matemática elementar apresentada pelos alunos.

As aulas presenciais no IFNMG-Campus Montes Claros foram suspensas no dia 18 de março de 2020 por causa da pandemia do novo coronavírus. Nesse contexto, foi necessária a adaptação da disciplina de Cálculo I para a modalidade ANP (Atividades Não Presenciais), permitida devido à situação de excepcionalidade do momento. $O$ engajamento dos alunos e dos professores, a organização do tempo e do espaço, aliada à autonomia, disciplina e organização apresentaram-se como novos desafios 
(CRAWFORD et al., 2020), além dos impostos pela própria disciplina. Somam-se a isso as limitações tecnológicas, o ambiente domiciliar, a pouca interação dos alunos entre si com os professores (DOSEA et al., 2020; SANDARS et al., 2020). Neste trabalho, apresentamos um panorama geral do curso de Pré-Cálculo seguido da disciplina Cálculo I ANP, ofertada aos ingressantes nos cursos de Engenharias e Ciência da Computação do IFNMG-Campus Montes Claros em 2020, bem como uma breve análise do desenvolvimento do ensino e seus resultados. Refletimos, ainda, sobre novos caminhos a seguir no intuito de melhorar as próximas ofertas desta disciplina.

\section{METODOLOGIA}

No sentido de dirimir a reprovação e evasão na disciplina de Cálculo 1, implementamos um curso de Pré-cálculo para os ingressantes dos cursos de Engenharia Elétrica, Engenharia Química e Ciência da Computação no IFNMG-Campus Montes Claros. A proposta inicial seria oferecer um curso de 80 horas, semipresencial, com aulas expositivas presenciais sobre os conteúdos do ensino básico, estudos autônomos através de resolução de exercícios a distância e encontros semanais presenciais com monitores para resolução de exercícios e esclarecimento de dúvidas.

Com o objetivo de selecionar os candidatos-bolsistas para atuarem como monitores no curso de formação continuada de Pré-Cálculo, publicou-se um edital tendo como público-alvo discentes dos cursos de graduação do Instituto Federal do Norte de Minas Gerais - Campus Montes Claros que tenham cursado a disciplina de Cálculo $1 \mathrm{com}$ aprovação. Foram disponibilizadas 13 vagas de monitores, sendo 12 na modalidade bolsista 1(atuando na elaboração e produção de material didático, estudos e atendimento ao público) e uma vaga na modalidade bolsista 2 (atuando como colaborador na estrutura e condução do curso junto aos docentes responsáveis). A seleção desses monitores ocorreu em duas etapas, sendo a primeira etapa eliminatória constituída por uma prova de conhecimentos básicos de matemática e a segunda etapa, classificatória, uma entrevista em que foi avaliado a capacidade de comunicação e expressão do candidato e sua disponibilidade.

Visando a capacitação dos monitores selecionados para que os mesmos tivessem o embasamento teórico necessário para acompanhar e auxiliar o docente em atividades relacionadas ao componente curricular, foi disponibilizado uma apostila como leitura suplementar, contendo 85 questões abrangendo todo o conteúdo que seria abordado no curso (essa apostila foi trabalhada com os alunos ao decorrer do curso). Ademais, ministrou-se aulas com os monitores para obliterar as possíveis dúvidas sobre o conteúdo antes do início do curso.

O curso de Pré-Cálculo foi oferecido como curso de Formação Inicial e Continuada (FIC), não sendo obrigatório mas altamente recomendado. Tivemos um total de 76 alunos matriculados e o curso aconteceu durante as três primeiras semanas do semestre letivo de 2020 (meses de fevereiro e março), sendo a última semana concomitante ao início da disciplina de Cálculo 1.

No entanto, quando as aulas presenciais foram suspensas devido à pandemia de COVID-19, o IFNMG passou a se organizar para o retorno das aulas como atividades não presenciais, tendo o primeiro semestre de 2020 sendo adiado para setembro. Neste período, a assistência estudantil fez levantamento dos alunos sem recursos para acompanhar as aulas de forma remota enquanto professores e coordenação se organizavam para retomar as disciplinas. Neste período, realizamos uma enquete com os alunos matriculados no Cálculo I para saber quantos teriam interesse em fazer um curso de Pré-Cálculo a distância e tivemos a adesão de $97 \%$ dos respondentes. Assim, de maio 
a agosto, oferecemos outro curso de Pré-Cálculo para 160 alunos, totalmente à distância, com um total de 100 horas. A disciplina regular de Cálculo 1 foi ofertada de setembro a dezembro de 2020, como atividade não presencial (ANP).

Tanto para o curso de Pré-Cálculo quanto para a disciplina de Cálculo 1 foram criadas salas de aula virtual no Google Classroom. Nelas, foram disponibilizados o cronograma, plano de ensino, atividades assíncronas, link para encontros síncronos, gravações dos encontros síncronos, listas de exercícios, avaliações, videoaulas e fóruns para dúvidas. Foram utilizadas videoaulas da plataforma do portal da Olimpíada Brasileira de Matemática para Escolas Públicas (OBMEP) (https://portaldaobmep.impa.br/index.php/site/index?a=1) para as aulas de Pré-Cálculo e da UNIVESP-TV (http://univesptv.com.br/ ), ambas de livre acesso, com aulas expositivas sobre os conteúdos. Semanalmente foram realizados dois encontros síncronos (gravados e disponibilizados posteriormente) para explicação de conteúdos, dúvidas e resolução de exercícios.

Os dois cursos à distância tiveram o apoio de monitores que ficaram responsáveis por acompanhar e auxiliar os alunos, resolvendo exercícios e esclarecendo dúvidas via WhatsApp ou Google Meet. O curso de Pré-Cálculo contou com oito monitores voluntários, sendo que a maioria destes monitores participaram como bolsistas no curso de formação continuada de Pré-Cálculo presencial anteriormente. Já o Cálculo I, contou com 2 monitores bolsistas.

Durante o desenvolvimento do curso de Pré-Cálculo e da disciplina de Cálculo 1, acompanhamos os alunos através do Google Classroom e de questionários do Google Formulários. Os resultados mais relevantes serão abordados na próxima seção.

\section{RESULTADOS E DISCUSSÃO}

Com o objetivo de avaliar como os alunos conduziram seu processo de aprendizagem para as atividades não-presenciais (ANPs) do curso de Cálculo I, e, também, para conhecer o perfil dos alunos, elaboramos um questionário utilizando o Google Form, que foi disponibilizado para os alunos através da Google Classroom. A seguir, apresentamos os resultados mais relevantes dessa pesquisa.

A maioria dos matriculados $(71,5 \%)$ são oriundos de escolas públicas. Além disso, $65 \%$ dos alunos declararam que concluíram o Ensino Médio a um ano ou mais. Esses fatores podem contribuir para o aumento das lacunas em relação ao conhecimento matemático básico, pois o tempo afastado das escolas e a fragilidade apresentada pelo ensino público são coeficientes importantes a serem analisados.

Com relação à forma de entrada na instituição, a porcentagem de alunos que entrou pelo sistema de cotas e pela ampla concorrência foi a mesma. Além disso, 12,3\% dos alunos ingressaram no IFNMG via transferência de outras instituições ou eram portadores de diploma. Assim, podemos considerar que este fator não teria tanta influência em nossa pesquisa.

Com relação a experiências anteriores com ensino remoto ou educação a distância, $78,8 \%$ dos alunos afirmaram não ter nenhuma experiência com esta modalidade antes da pandemia de COVID-19, o que dificulta a sua adaptação em tal modalidade de ensino. Além disso, $44,7 \%$ dos respondentes afirmaram não possuir um ambiente adequado para estudo durante as disciplinas ANP. Diante da dinâmica de aprendizagem promovida pelo ensino a distância tornou-se necessário ajustar rotinas pessoais com os estudos, separando espaço e tempo para ambos, afetando a autonomia do aluno para estudar. Porém, mesmo com todas as dificuldades elencadas, a maioria 
dos alunos afirmou conseguir se organizar e cumprir a rotina de estudos durante a semana.

Uma das questões elencadas no questionário referia-se ao conhecimento prévio de matemática e seus desafios na aprendizagem de Cálculo. Segundo os respondentes, fatores como falta de concentração, compreensão de novos conceitos e a fragilidade com relação à matemática básica são os que mais afetam o aprendizado de Cálculo I. Com relação à matemática do ensino básico, os temas que mais despertam dificuldade entre os alunos são trigonometria, seguido de funções. É importante destacar também que o meio para sanar dúvidas mais usado pelos alunos foi a internet, seguido dos colegas e, só então, dos professores. Além disso, um dos menores índices nesse aspecto da pesquisa foi a consulta aos monitores.

Quanto à presença dos alunos nos encontros síncronos, $22.3 \%$ dos alunos declararam que sempre acompanham ao vivo, $67.7 \%$ disseram que quando não podem acompanhar, assistem às gravações e $10 \%$ não assistem nem à gravação do encontro. Nota-se, portanto, que as aulas têm apelo entre os alunos, mesmo neste modelo à distância, mostrando que os mesmos ainda as consideram importantes neste momento.

Também os alunos foram questionados acerca do tempo que disponibilizam para os estudos de Cálculo I além do tempo de aula. Os dados estão dispostos na Figura 1 a seguir.

Figura 1 - Quantidade de horas de estudo semanal da disciplina de Cálculo I fora do horário de aula

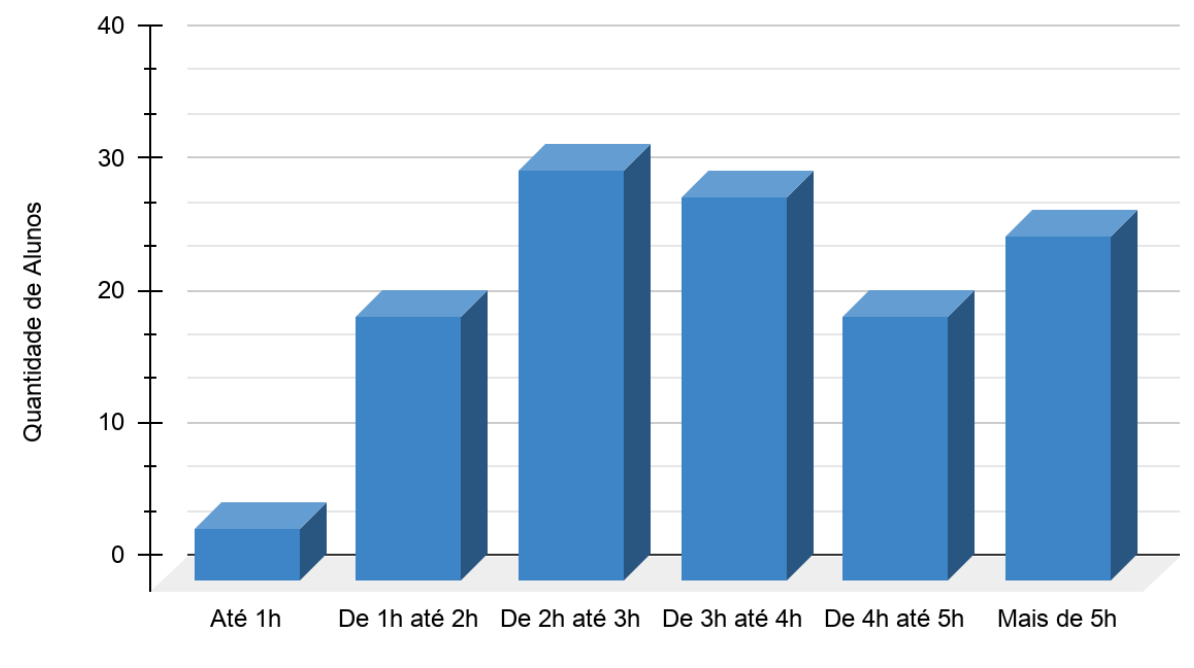

Número de horas de estudo semanais

Fonte: Arquivo Pessoal (2021)

Sendo o Cálculo ANP uma disciplina totalmente a distância, com carga horária de encontros presenciais e avaliações ocupando cerca de $65 \%$ do total, $35 \%$ da carga horária restante deveriam ser dedicados a atividades autônomas. Numa disciplina de 90 horas, isso corresponde a 31,5 horas. O curso teve duração de 13 semanas, o que corresponde a 2,5 horas para cumprimento de atividades autônomas como assistir os vídeos sugeridos, leitura de livros e resolução de exercícios. O plano de estudos, assim como a gestão do tempo e maneira como se aprende, é individual, não havendo fórmula eficaz que atenda a todos. Mesmo assim, observamos que o tempo de estudos dedicado ao cálculo tem média acima de 3 horas, o que pode ser considerado um tempo bom. Isto é importante no cenário em que vivemos, que exige mais esta disciplina e estudos fora do horário de aula. 
Podemos perceber a partir dos dados coletados, que, de modo geral, os alunos se mostraram mais independentes na questão do aprendizado pós-aula, destacando-se a autonomia e organização dos alunos, que dependem cada vez menos da figura do professor/monitor. Contudo, ainda temos problemas que haviam na modalidade presencial, como a dificuldade em conteúdos básicos de matemática, que tradicionalmente são um percalço na trajetória do aluno no curso de Cálculo I.

Com o objetivo de investigar a eficiência da aplicação das ANPs e reelaborar a metodologia para uma aplicação futura, buscamos os índices de aprovação, dispostos na Figura 2, obtidos de 2016 à 2020 na disciplina presencial de Cálculo I nos cursos de Ciência da Computação e Engenharia Química.

Figura 2 - Índices, em porcentagem, de aprovação da disciplina de Cálculo I no Instituto Federal do Norte de Minas Gerais (IFNMG), campus Montes Claros entre 2016 e 2020.

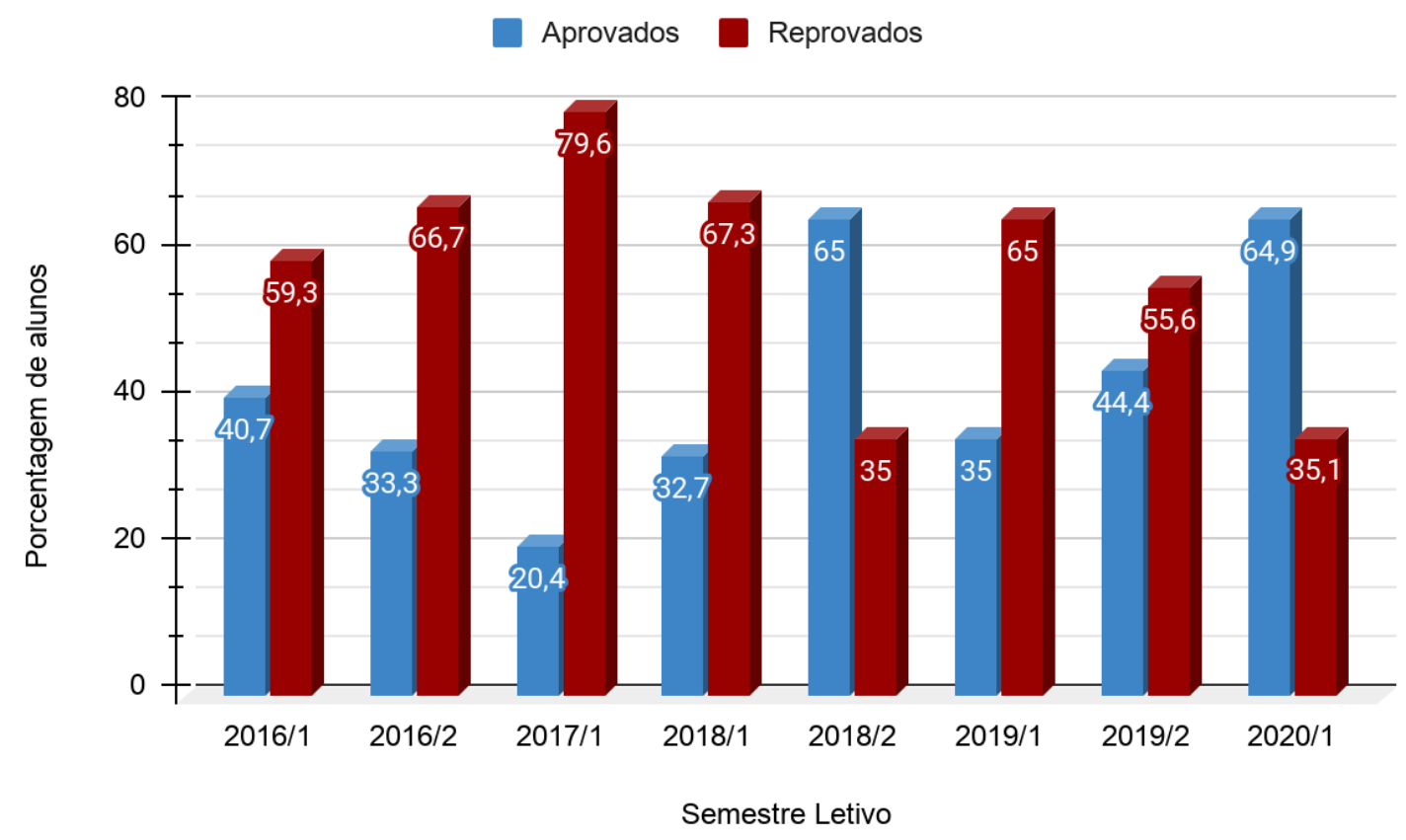

Fonte: Arquivo Pessoal (2021).

É importante ressaltar que ao compararmos os valores obtidos nos anos anteriores (2016 a 2019) e no ano de 2020, com as aulas online, houve um aumento expressivo no número de aprovações, subindo, em média, de $36 \%$ para $65 \%$.

Salienta-se que cerca de $70 \%$ dos matriculados no Cálculo I cursaram o curso de Formação Inicial e Continuada (FIC) de Pré-Cálculo. Sendo assim, solicitamos que os alunos desta disciplina respondessem um formulário para avaliação do curso de Pré-Cálculo, com o objetivo de coletar dados e investigar a eficiência do Pré-Cálculo para o acompanhamento da disciplina de Cálculo I e reelaborar a metodologia para uma aplicação futura. A seguir, os resultados dessa pesquisa.

Como o questionário foi disponibilizado após o encerramento da disciplina de Cálculo I, tivemos retorno da metade dos alunos matriculados. De acordo com o formulário, $83 \%$ afirmam que o Pré-Cálculo colaborou com a aprovação, dito como essencial $(47 \%)$ ou muito importante $(36 \%)$. Estes dados ratificam a necessidade da inclusão de cursos de complementação deste nível para os ingressantes, ou, possivelmente, a inclusão de disciplinas na matriz curricular dos cursos superiores que 
visam contemplar as necessidades de conhecimentos matemáticos básicos. Essas medidas podem colaborar efetivamente com a redução dos altos índices de evasão.

Ademais sendo os três conteúdos julgados por eles como mais importantes: funções, trigonometria e álgebra. Os alunos encaminharam sugestões para melhoria do curso, dos quais destacam-se: mais enfoque em trigonometria, mais encontros com os monitores e maior quantidade de exercícios.

Os alunos que não cursaram o Pré-Cálculo (19\%) e responderam ao formulário, quando questionados por qual motivo não participou do curso, justificaram com a falta de tempo/horário incompatível; não ter julgado importante ou já ter uma boa base matemática. Porém, $71,4 \%$ destes afirmaram ter sentido falta de ter cursado o Pré-Cálculo para acompanharem a disciplina de Cálculo I, sendo os conteúdos que mais sentiram falta de revisão: trigonometria, álgebra, funções, matrizes, sistemas e determinantes.

Perante o exposto, destacamos aqui a necessidade que os alunos apresentam em aprofundar conhecimentos matemáticos básicos para o bom acompanhamento das disciplinas dos cursos superiores na área de exatas. As lacunas incidem nos altos índices de reprovação e/ou abandono na disciplina de Cálculo 1, o que contribui para evasão do curso. Assim, entendemos que o oferecimento do curso de Pré-Cálculo é uma medida contra evasão trazendo para o aluno, além de conhecimentos teóricos, aumento da autoconfiança e melhor entrosamento com o curso.

No entanto, não podemos atribuir os altos índices de aprovação ao curso de Pré-Cálculo, uma vez que vivenciamos um período atípico, no qual o Cálculo 1 foi oferecido na modalidade não presencial emergencial devido à pandemia, podendo haver outros fatores relevantes que não foram analisados neste trabalho, como, por exemplo, os métodos de avaliação adotados no período ANP. Como as atividades avaliativas nessa modalidade foram realizadas online, perguntou-se aos alunos, através do questionário anônimo de autoavaliação, sobre a ética no cumprimento dessas atividades. A maioria dos alunos declarou que não se vale de ajudas externas nestes momentos. Ademais, $72 \%$ dos alunos consideraram seu nível de aprendizado na disciplina como ótimo ou excelente e $64 \%$ afirmam que se sentem preparados para cursar Cálculo II com mérito.

Por fim, os alunos citaram como fatores que podem contribuir para a melhoria da disciplina de Cálculo I: uso de aplicações do conteúdo para incentivar o aprendizado, utilização de técnicas como brainstorming, discussões em grupos sobre questões, melhor fiscalização nas avaliações, aumento do número de aulas de resolução de exercícios, maior utilização de softwares como Geogebra e oferecimento de curso de nivelamento como o pré-cálculo. Sem dúvidas, os aspectos considerados são relevantes e permitem traçar novas diretrizes para reformulação da disciplina.

\section{CONSIDERAÇÕES FINAIS}

Neste trabalho, foi feita uma descrição de como foi executado o curso de Cálculo I ANP e os métodos utilizados no mesmo. A partir de dados coletados por meio de um questionário feito com os alunos, foram feitas análises de como procedeu a aplicação da modalidade não presencial e como esta afetou o desempenho e o aprendizado dos matriculados. Concluiu-se que, apesar das dificuldades impostas a todos os agentes participantes do ensino, o curso se desenvolveu bem, atingindo uma boa porcentagem de aprovação dos seus participantes, com os alunos mostrando um bom nível de independência na aprendizagem, sem perder a qualidade do ensino.

Destacamos, ainda, que o curso de Formação Intensiva e Continuada (FIC) de Pré-Cálculo foi de grande importância para o acompanhamento e desenvolvimento dos 
estudos no curso de Cálculo I dos alunos participantes. Além disso, baseando-se nas análises dos resultados obtidos em 2020 juntamente com as sugestões dos alunos foi possível reelaborar a metodologia para uma aplicação futura.

As lacunas de matemática herdadas no ensino básico se apresentam como percalços na condução do curso superior para os alunos de exatas e buscar estratégias para supri-las tem sido um desafio para toda a comunidade acadêmica. Neste contexto, seguimos na busca de alternativas para assegurar que o estudante supere as dificuldades e tenha mérito em sua carreira acadêmica.

Por fim, consideramos que o ensino remoto apresenta vantagens como desenvolvimento da autonomia do aluno, flexibilidade de horários, permitindo que tenha liberdade para estudar em horários mais adequados à sua realidade. No entanto, destacamos como desvantagens a falta de contato na sala de aula que diminui a relação interpessoal, os métodos de avaliação que não foram adequadamente adaptados para o ensino remoto, somando-se, ainda, os desafios enfrentados por docentes e discentes que tiveram que se adaptar de modo abrupto às novas metodologias e suas novas rotinas. Ainda assim, podemos concluir que houve mérito no processo, surgindo, inclusive, novas possibilidades anteriormente não vislumbradas.

\section{Agradecimentos}

Agradecemos ao Instituto Federal do Norte de Minas Gerais (IFNMG), Campus Montes Claros, pelo apoio financeiro durante o desenvolvimento deste projeto.

\section{REFERÊNCIAS.}

\section{Artigos de periódicos:}

AMBIEL, Rodolfo AM. Construção da escala de motivos para evasão do ensino superior. Avaliação Psicológica, v. 14, n. 1, p. 41-52, 2015.

DOSEA, Giselle Santana et al. Métodos ativos de aprendizagem no ensino online: a opinião de universitários durante a pandemia de COVID-19. Interfaces Científicas-Educação, v. 10 , n. 1, p. 137-148, 2020. DOl: https://doi.org/10.17564/2316-3828.2020v10n1p137-148.

SILVA FILHO, R. L. L. et al. A Evasão no Ensino Superior Brasileiro. Caderno de Pesquisa, v. 37, n. 132, p. 641-659, set./dez. 2007.

\section{Monografias, dissertações e teses:}

BARBOSA, M. A. 0 insucesso no ensino e aprendizagem na disciplina de cálculo diferencial e integral. 2004. 102f. Dissertação (Mestrado em Educação) - Pontifícia Universidade Católica do Paraná, Curitiba, 2004. Disponível em: http://www.biblioteca.pucpr.br/tede//tde busca/arquivo.php?codArquivo=291. Acesso em: 09 maio. 2021.

BARDAGI, M. P. Evasão e Comportamento Vocacional de Universitários: estudos sobre o desenvolvimento de carreiras na graduação. 2007. 242 p. Tese (Doutorado em Psicologia) - Universidade Federal do Rio Grande do Sul, Rio Grande do sul, 2007. Disponível em: https://www.lume.ufrgs.br/handle/10183/10762. Acesso em: 09 maio. 2021. 
GOMES, K. A. Indicadores de permanência na educação superior : o caso da disciplina de cálculo diferencial integral I. 2015. 217f. Dissertação (Mestrado em Educação) - Centro Universitário La Salle, Canoas, 2015. Disponível em: http://svr-net20.unilasalle.edu.br/handle/11690/620. Acesso em: 09 maio. 2021.

GAIOSO, N. P. L. O fenômeno da evasão escolar na educação superior no Brasil. Relatório técnico. Pró-reitoria de Pós-graduação e Pesquisa, Universidade Católica de Brasília, 2005.

GILIOLI, Renato de Souza Porto. Evasão em instituições federais de ensino superior no Brasil: expansão da rede, SISU e desafios. Estudo Técnico - Câmara dos Deputados, Brasília: Câmara dos Deputados, 2016. Disponível em: https://bd.camara.leg.br/bd/bitstream/handle/bdcamara/28239/evasao_instituicoes_gilioli.p df? sequence=1. Acesso em: 08 maio. 2021.

\section{Artigos de jornais:}

CRAWFORD, J. et al.. Covid-19: 20 countries' higher education intra-period digital pedagogy responses. Journal of Applied Learning \& Teaching, v. 3, n. 1, 2020. DOI: https://doi.org/10.37074/jalt.2020.3.1.7. Acesso 10 abr. 2021.

SANDARS, John et al. Twelve tips for rapidly migrating to online learning during the COVID-19 pandemic. MedEdPublish, v. 9, n. 1,p.82, 2020. DOI: https://doi.org/10.15694/mep.2020.000082.1. Acesso em: 08 maio. 2021.

\section{Trabalhos em eventos:}

BARCELOS, Thiago Schumacher; SILVEIRA, Ismar Frango. Pensamento computacional e educação matemática: Relações para o ensino de computação na educação básica. In: XX Workshop sobre Educação em Computação, 2012, Curitiba. Anais do XXXII CSBC. Curitiba.

Disponível

em: http://www2.sbc.org.br/csbc2012/anais csbc/eventos/wei/artigos/Pensamento\%20Comput acional $\% 20$ e $\% 20$ Educacao $\% 20$ Matematica $\% 20$ Relacoes $\% 20$ para $\% 200 \% 20$ Ensino $\% 20 \mathrm{~d}$ e\%20Computacao\%20na\%20Educacao\%20Basica.pdf. Acesso em 08 maio. 2021.

KESSLER, M. C. Em busca da diminuição dos índices de evasão no cálculo diferencial: a experiência da UNISINOS. In: Congresso Brasileiro de Educação em Engenharia, 2013, Gramado. Anais do XLI Congresso Brasileiro em Educação em Engenharia. Disponível em: https://www.turing.pro.br/anais/COBENGE-2013/pdf/117863 1.pdf. Acesso em 08 maio. 2021.

WROBEL, Julia Schaetzle; ZEFERINO, Marcus Vinicius Casoto; CARNEIRO, Teresa Cristina Janes. Um mapa do ensino de Cálculo nos últimos 10 anos do COBENGE. In: Congresso Brasileiro de Educação em Engenharia, 2013, Gramado. Anais do XLI Congresso Brasileiro em Educação em Engenharia. Disponível em: https://www.turing.pro.br/anais/COBENGE-2013/pdf/117437_1.pdf. Acesso em 08 maio. 2021. 


\title{
OVERVIEW OF NON-PRESENTIAL ACTIVITIES RELATING TO PRE-CALCULUS AND CALCULUS I AT IFNMG - CAMPUS MONTES CLAROS
}

\begin{abstract}
Many students start classes in college education with significant gaps in relation to math knowledge (GOMES, 2012). Therefore, it's considerable that many ideas were misunderstood, which makes it more difficult to learn new concepts. The subjects of Differential and Integral Calculus presents high failure rates in the courses of INMGCampus Montes Claros, and in other institutions, too. By the way, the low performance in these subjects results in the discouragement of the students that sometimes choose to give up the course. The absence from basic elementary mathematics contributes significantly to this scenario. According to this, it is necessary to develop projects and programs aimed at combating repetition and dropout in these subjects, making successful students, in addition to establishing solid mathematical knowledge. This study explored an overview of the Pre-Calculus course according to the Calculus I ANP course, that was offered to Engineering and Computer Science students who courses at the IFNMGCampus Montes Claros in 2020, like a way to analyze the development of teaching and the results. Finally, it was reflected by new ways to follow, trying to improve the next apprenticeship in these subjects.
\end{abstract}

Keywords: Teaching. Non-face-to-face activities. Calculus. Basic math. 